Noname manuscript No.

(will be inserted by the editor)

\title{
Discovery of Accessible Locations using Region-based Geo-social Data
}

\author{
Yan Wang · Jianmin Li · Ying Zhong • \\ Shunzhi Zhu* . Danhuai Guo • \\ Shuo Shang*
}

Received: / Accepted:

\begin{abstract}
Geo-social data plays a significant role in location discovery and recommendation. In this light, we propose and study a novel problem of discovering accessible locations in spatial networks using region-based geo-social data. Given a set $Q$ of query regions, the top- $k$ accessible location discovery query $(k \mathrm{ALDQ})$ finds $k$ locations that have the highest spatial-density correlations to $Q$. Both the spatial distances between locations and regions and the POI (point of interest) density within the regions are taken into account. We believe that this type of $k$ ALDQ query can bring significant benefit to many applications such as travel planning, facility allocation, and urban planning. Three challenges exist in $k$ ALDQ: (1) how to model the spatial-density correlation practically, (2) how to prune the search space effectively, and (3) how to schedule the searches from multiple query regions. To tackle the challenges and process $k$ ALDQ effectively and efficiently, we first define a series of spatial and density metrics to model the spatial-density correlation. Then we propose a novel three-phase solution with a pair of upper and lower bounds of the spatial-density correlation and a heuristic scheduling strategy to sched-

\footnotetext{
* Corresponding Authors: Shunzhi Zhu and Shuo Shang

Yan Wang • Jianmin Li • Ying Zhong • Shunzhi Zhu

Xiamen University of Technology, China

E-mail: \{wangyan,lijm,yzhong,szzhu\}@xmut.edu.cn

Guangdong Province Key Laboratory of Popular High Performance Computers of Shenzhen University, and Guangdong Provincial Big Data Collaborative Innovation Center, Shenzhen University

CNIC, Chinese Academy of Sciences, Beijing, China

E-mail: guodanhuai@cnic.cn
}

Danhuai Guo

Shuo Shang

King Abdullah University of Science and Technology, Saudi Arabia

E-mail: jedi.shang@gmail.com
\end{abstract}


ule multiple query regions. Finally, we conduct extensive experiments on real and synthetic spatial data to demonstrate the performance of the developed solutions.

Keywords Location Discovery $\cdot$ Region $\cdot$ Recommendation · Geo-social Data

\section{Introduction}

With the rapid development of GPS-equipped smart phones and online-map services (e.g., Google-maps ${ }^{1}$, Baidu-maps ${ }^{2}$, and MapQuest ${ }^{3}$ ), people can easily get their current geographic locations and post geo-tagged tweets, geo-tagged photos, and geo-tagged checkins to specialized sites (e.g., twitter ${ }^{4}$, flickr $^{5}$, and foursquare $^{6}$ ). Such massive geo-social data enable many novel application. An emerging one is region-based accessible location discovery. Given a set $Q$ of query regions, the top- $k$ accessible location discovery query ( $k$ ALDQ) finds $k$ locations that have the highest spatial-density correlations to $Q$. We believe that this type of $k$ ALDQ query can bring significant benefit to many applications such as travel planning, facility allocation, and urban planning.

In most of existing studies $[21,22,25,27,36]$, location discovery and recommendation is based on point-to-point matching $[21,22,25]$ or trajectory-topoint matching $[27,36]$. They only take the spatial distances between matched points or matched trajectories and points into account, but no existing study considers the regions of interest and the density of spatial objects (e.g., geotagged tweets, geo-tagged photos, and POIs) in the corresponding regions. For example, there exist several regions of interest (e.g., residential area, commercial area, or scenic area), and we want to find an aggregate location for travelers, or we want to find an accessible location to set a new facility (e.g., shopping mall, bank, or petrol station) to maximize its commercial value. The accessible locations are expected spatially close to the regions of interest. In particular, they are expected spatially close to the regions with high spatialobject density.

An example is shown in Figure 1 , where $R_{1}, R_{2}$, and $R_{3}$ are query regions, $p_{1}$ and $p_{2}$ are location candidates, and the red points are spatial objects. If we only consider the spatial distances between location and query regions (i.e., $d\left(c_{1}, p_{1}\right)+d\left(c_{2}, p_{1}\right)+d\left(c_{3}, p_{1}\right)$, where $c_{1}, c_{2}$, and $c_{3}$ are center points of $R_{1}, R_{2}$, and $R_{3}$ ), location $p_{1}$ is returned because it is spatially close to $R_{1}, R_{2}$, and $R_{3}$. However, if we take both spatial distance and the density of spatial objects in the query regions into account, location $p_{2}$ will be returned. Although $p_{2}$ is not as good as $p_{1}$ in the spatial domain, $p_{2}$ is spatially close to the regions

\footnotetext{
1 http://maps.google.com/

2 http://map.baidu.com/

3 http://www.mapquest.com

4 https://twitter.com/

5 https://www.flickr.com/

6 https://foursquare.com/
} 


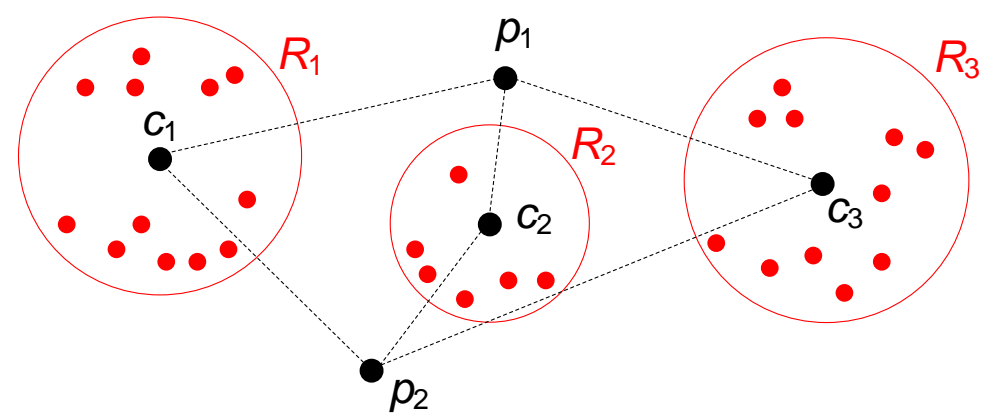

Fig. 1 An example of $k$ ALDQ

with high spatial-object density, so we still consider $p_{2}$ as the optimal choice. To the best of our knowledge, this is the first study of location discovery and recommendation that take both spatial distance and spatial-object density into account.

$k$ ALDQ is conducted in spatial networks, because in most practical scenarios, objects move in spatial networks (e.g., road networks) rather than in Euclidean space. The existing techniques of location discovery and recommendation cannot compute $k$ ALDQ due to three reasons. First, $k$ ALDQ considers the matching between regions and locations, rather than the point-to-point matching or trajectory-to-point matching, thus it needs new distance metrics. Second, $k$ ALDQ takes the density of spatial objects in query regions into account, so it also needs specific metrics to reflect the density domain. Third, most existing studies of aggregate location query [21,22] are conducted in Euclidean space, and their optimization techniques cannot be used in spatial networks directly, because most spatial indexes (e.g., R-tree) use Euclidean space as lower bound to prune the search space and such bound is ineffective in spatial networks.

$k$ ALDQ faces three challenges. First, it needs a metric to describe spatialdensity correlation. Second, it needs a pair of comparable tight upper and lower bounds of the spatial-density correlation to prune the search space. Third, it needs a heuristic scheduling methods to schedule multiple query regions to further enhance the query performance. To overcome the challenges and to process $k$ ALDQ efficiently, we propose a novel spatial-density correlation measure. Then we develop a novel three phase algorithm. In the location-search phase, we expand network expansions according to Dijkstra's algorithm [7] to explore the spatial network and to find location candidates. A heuristic scheduling method is defined to schedule the search processes of different query regions. In the filter phase, we define a pair of upper and lower bounds of the spatial-density correlation to prune the search space. Once the global lower bound exceeds the global upper bound, the search terminates and all partly and unscanned locations are pruned. In the refine phase, we compute the 
exact spatial-density correlations for all full scanned locations and return top$k$ results.

To sum up, we make the following contributions in this paper.

- We propose a novel type of top- $k$ accessible location discovery query ( $k$ ALDQ) to find top- $k$ accessible locations in a spatial network. We believe that this type of query can bring significant benefit in many applications such as travel planning, facility allocation, and urban planning.

- We define a series of new metrics to evaluate the spatial and density correlations between regions and locations (Section 2).

- We develop a range-query based baseline algorithm to process $k$ ALDQ (Section 3.1).

- We develop a novel three phase algorithm to process $k$ ALDQ efficiently with the support of a pair of upper and lower bounds and a heuristic scheduling method (Section 3.2).

- We conduct extensive experiments on real and synthetic spatial data to demonstrate the performance of the developed algorithms (Section 4).

The rest of the paper is organized as follows. The spatial networks and the metrics of the spatial-density correlation as well as problem definitions are introduced in Section 2. The three-phase algorithm is introduced in Section 3 , which is followed by extensive experimental results in Section 4. Related work is covered in Section 5 and conclusions and future directions are given in Section 6.

\section{Preliminaries}

2.1 Spatial Networks, Locations, Spatial Objects, and Regions

A spatial network $G(V, E)$ is modeled by a connected and undirected graph, where $V$ is a vertex set and $E \subseteq V \times V$ is an edge set. The weight $w(e)$ of an edge $e \in E$ represents its weight (e.g., distance or travel time). We use adjacent list to store spatial networks.

Generally, locations and spatial objects are of the form of (longitude, latitude). We map these locations and spatial objects to the corresponding spatial network by using some existing map-matching method [1]. We assume that all locations and spatial objects are on vertices. It is straightforward to support the scenarios that locations and spatial objects are on edges. Assuming that a location $p$ is on an edge $(a, b)$ and the network distance from $p$ to $a$ and $b$ are known. We can create a new vertex $p$ and split edge $(a, b)$ into $(a, p)$ and $(p, b)[26]$.

We align the definitions of spatial networks, locations, and spatial objects with existing studies [24-26, 29,37].

Given two vertices $a$ and $b$ in a spatial network, the network shortest path distance between $a$ and $b$ is denoted by $s d(a, b)$. A region $R$ in spatial networks is a circular area $(c, r)$, where $c$ is a center point of $R$ and $r$ is the radius. For 
all spatial objects whose network distances to $c$ do not exceed $r$, we say that they are contained by region $R$.

\subsection{Metrics}

Given a location $p$ and region $R$, the spatial-density correlation between $p$ and $R$ is defined as follows.

$$
C_{S D}(p, R)=\sum_{o \in R} e^{-s d(p, o)}
$$

Here, $o$ is the spatial object in region $R$, and we consider the aggregate distance between $p$ and all objects in $R$. Given a set of query regions $Q=$ $\left\{R_{1}, R_{2}, \ldots, R_{n}\right\}$, and a location $p$, the spatial-density correlation between $p$ and $Q$ is defined as follows.

$$
C_{S D}(p, Q)=\sum_{R_{i} \in Q}\left(\frac{2}{1+e^{-C_{S D}\left(p, R_{i}\right)}}-1\right)
$$

Here, we use the Sigmoid function $[26,28]$ to normalize $C_{S D}\left(p, R_{i}\right)$ to a range $[0,1]$, and each query region plays an equal role in query processing.

\subsection{Problem Definition}

Given a set of query regions $Q$, and a set of location candidates $P$, the top- $k$ accessible location discovery query ( $k$ ALDQ) finds a $k$-item set $A(|A|=k)$ that contains $k$ locations with the highest spatial-density correlations to $Q$, such that $\forall p^{\prime} \in P \backslash A\left(p \in A\left(C_{S D}\left(p^{\prime}, Q\right) \leq C_{S D}(p, Q)\right)\right)$.

\section{Query Processing}

\subsection{Baseline Method}

Range query is a straightforward baseline method to $k$ ALDQ. From each query region $R_{i}$, we expand the search from its center $c_{i}$ at the same speed by increasing $r s_{i}$ as $r s_{i}=r s_{i}+x$, where $r s_{i}$ is the search radius of query region $R_{i}$ and $x$ is a user given parameter. An example is shown in Figure 2, where $R_{1}$ and $R_{2}$ are two query regions, $c_{1}$ and $c_{2}$ are the centers, $r_{1}$ and $r_{2}$ are the radiuses of query regions, and $r s_{1}$ and $r s_{2}$ are the search radiuses.

For a location candidate $p$, if it is scanned by the search from query region $R$ (e.g., $p_{1}$ and $R_{2}$ ), we estimate its spatial-density correlation $C_{S D}(p, R)$ as follows. According to the triangular inequality, for each spatial object $o \in R$, we have that

$$
\begin{aligned}
& s d(o, p)<s d(o, c)+s d(c, p) \\
& s d(o, p)>s d(c, p)-s d(o, c)
\end{aligned}
$$




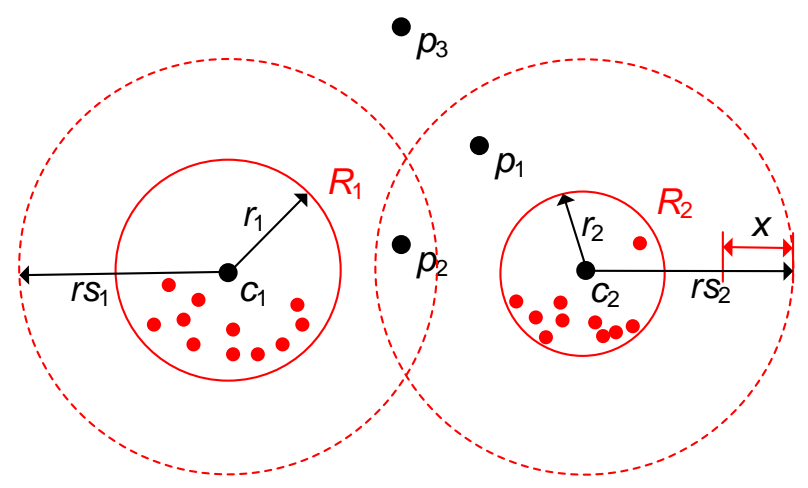

Fig. 2 An example of Baseline Algorithm

By substituting Equation 3 into Equation 1, we have the lower bound $C_{S D}(p, R) . l b$ of spatial-density correlation as follows.

$$
\begin{aligned}
s d(o, p) & <s d(o, c)+s d(c, p) \Rightarrow e^{-s d(o, p)}>e^{-(s d(o, c)+s d(c, p))} \\
C_{S D}(p, R) & =\sum_{o \in R} e^{-s d(p, o)}>\sum_{o \in R} e^{-(s d(c, p)+s d(o, c))}=C_{S D}(p, R) \cdot l b
\end{aligned}
$$

If a location candidate is fully scanned (i.e., scanned by expansions from all query regions, e.g., $p_{2}$ in Figure 2), we estimate the lower bound of $C_{S D}(p, R)$ by substituting Equation 5 into Equation 2 .

$$
C_{S D}(p, Q) \cdot l b=\sum_{R_{i} \in Q}\left(\frac{2}{1+e^{-C_{S D}\left(p, R_{i}\right) \cdot l b}}-1\right)
$$

For unscanned location candidates (e.g., $p_{3}$ in Figure 2), we estimate the upper bound of their spatial-density correlations as follows. For an unscanned location candidate $p$, we have that $s d(c, p)>r$. By substituting it into Equations 4 and 1 , we have that

$$
\begin{gathered}
s d(o, p)>s d(c, p)-s d(o, c) \Rightarrow s d(o, p)>r-s d(o, c) . \\
C_{S D}(p, R)=\sum_{o \in R} e^{-s d(p, o)}<\sum_{o \in R} e^{-(r-s d(o, c))}=C_{S D}(p, R) . u b
\end{gathered}
$$

By substituting Equation 7 into Equation 2, the upper bound of $C_{S D}(p, Q)$ is defined as follows.

$$
C_{S D}(p, Q) \cdot u b=\sum_{R_{i} \in Q}\left(\frac{2}{1+e^{-C_{S D}\left(p, R_{i}\right) \cdot u b}}-1\right)
$$

The value of $C_{S D}(p, Q) . u b$ is the global upper bound of all unscanned location candidates (e.g., $p_{3}$ in Figure 2). Among all fully scanned location candidates, we define a global lower bound $L B$ as follows.

$$
L B=\min _{p \in P_{k}}\left\{C_{S D}(p, Q) . l b\right\}
$$




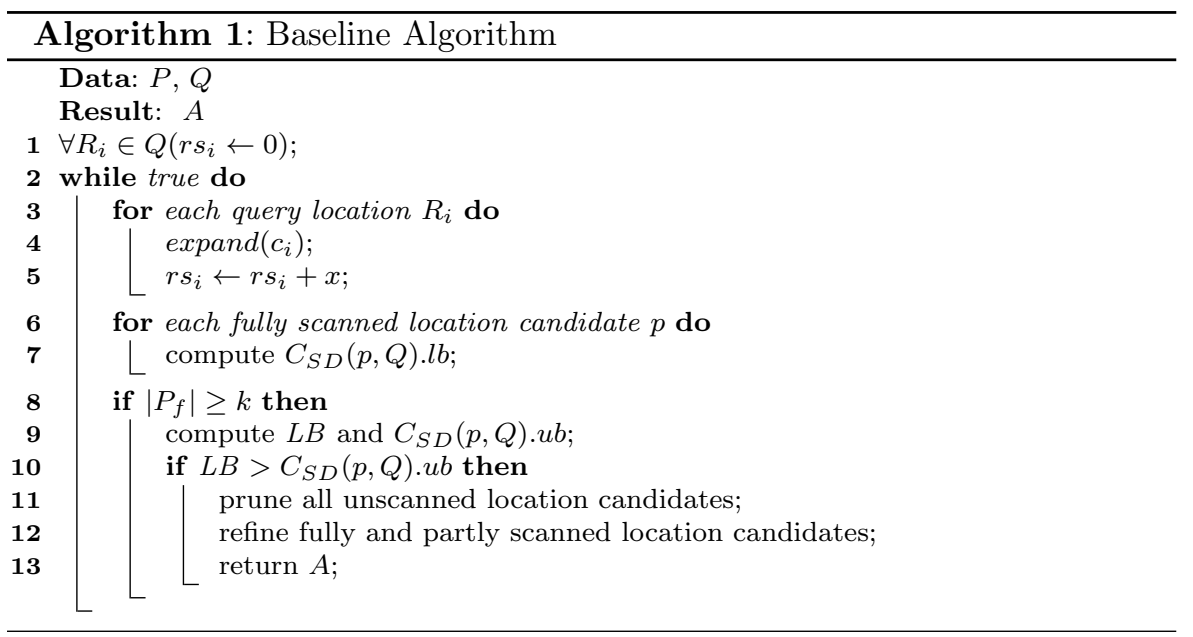

Here, $P_{k} \subseteq P_{f} \wedge\left|P_{k}\right|=k$, and $P_{f}$ is a set of all fully scanned location candidates and $P_{k}$ is a subset of $P_{f}$. Set $P_{k}$ contains $k$ items with the largest values of $C_{S D}(p, Q) . l b$, such that $\forall p \in P_{k}\left(\forall p^{\prime} \in P_{f} \backslash P_{k}\left(C_{S D}\left(p^{\prime}, Q\right) . l b<C_{S D}(p, Q) . l b\right)\right)$. The value of $L B$ changes continually during query processing.

Once the value of $C_{S D}(p, Q) . u b$ is less than that of $L B$, the searches from all query regions terminate, and all unscanned location candidates are pruned. Then, we continuously refine all fully scanned (e.g., $p_{2}$ in Figure 2) and partly scanned location candidates (e.g., $p_{1}$ in Figure 2). Among all refined location candidates, we select $k$ location candidates with the largest spatial-density correlation and return them.

The baseline algorithm is detailed in Algorithm 1. The query arguments include a set $P$ of location candidates and a set $Q$ of query regions. The query output is a set $A(|A|=k \wedge A \subseteq Q)$ of location candidates with largest spatial-density correlations. Initially, the search radiuses of each query region are set to 0 (line 1 ). We make the range query from each query region and expand the search as $r s_{i}=r s_{i}+x$ (line 2-5). For each fully scanned location candidate $p$, we compute its lower bound $C_{S D}(p, Q) . l b$ of spatial-density correlation (Equation 6). If the size of fully scanned set exceeds $k$, we compute the global lower bound $L B$ of fully scanned location candidates (Equation 9) and the upper bound $C_{S D}(p, Q) . u b$ of unscanned location candidates (Equation 8) (lines 8-9). If the value of $L B$ exceeds that of $C_{S D}(p, Q) . u b$, the searches from all query regions terminate, and all unscanned location candidates are pruned (lines 11-12). Then, we refine all fully scanned and partly scanned location candidates, and find top- $k$ locations in set $A$ with the largest spatial-density correlations (lines 13-14). 


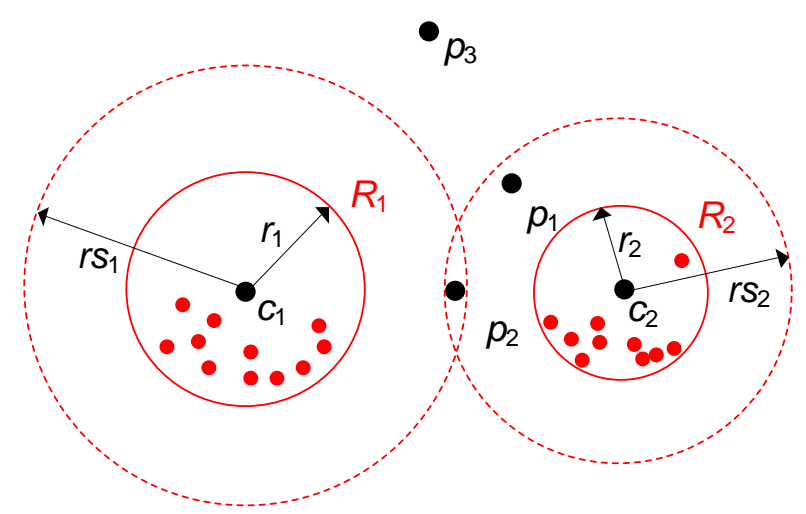

Fig. 3 An example of Three-Phase Algorithm

\subsection{Three-phase Algorithm}

The baseline algorithm includes several weaknesses. First, its search is based on range query, so it is not continuous. It is difficult to set the expansion parameter $x$. Second, it simply prunes the unscanned location candidates, and it has to refine all fully scanned and partly scanned location candidates. Its pruning power is comparable weak. Third, the expansion speed from each query region is the same, and it lacks an effective scheduling method to schedule the searches from multiple query regions.

To overcome these weaknesses, we propose a novel three phase algorithm to process the $k$ ALDQ query efficiently. In the location search phase, we adopt network expansion method [7] to explore the spatial network and to identify locations spatially close to the query regions. A pair of upper and lower bounds of the spatial-density correlation are defined to prune the search space. All partly scanned and unscanned location candidates are pruned. In the scheduling phase, we propose a heuristic scheduling method to schedule multiple query regions to further enhance the query efficiency. In the refinement phase, we refine all fully scanned location candidates and find the query result.

\subsubsection{Expansion and Bounds}

We conduct network expansion from each query region according to Dijkstra's algorithm [7] to explore the spatial network and find location candidates close to the query regions. An example is shown in Figure 3 , where $R_{1}$ and $R_{2}$ are two query regions, $c_{1}$ and $c_{2}$ are centers, and $r_{1}$ and $r_{2}$ are radiuses of regions $R_{1}$ and $R_{2}$. The explored regions are circular areas, and $r s_{1}$ and $r s_{2}$ are the corresponding radiuses (i.e., the network distance from center to the expansion boundary).

Similar to the baseline algorithm, the location candidates are classified into three categories, fully scanned (e.g., $p_{2}$ in Figure 3), partly scanned (e.g., $p_{1}$ ), and unscanned (e.g., $p_{3}$ ). If a location candidate $p$ is fully scanned, we estimate 
its lower bound of the spatial-density correlation according to Equation 6 . Among all fully scanned location candidates, we define a global lower bound according to Equation 9.

For a partly scanned location candidate $p$, we estimate its upper bound of the spatial-density correlation as follows. If $p$ is scanned by the expansion from $R$, we have that

$$
\begin{gathered}
s d(o, p)>s d(c, p)-s d(o, c) \\
\Rightarrow C_{S D}(p, R)=\sum_{o \in R} e^{-s d(p, o)}<\sum_{o \in R} e^{-(s d(c, p)-s d(o, c))}=C_{S D}(p, R) . u b
\end{gathered}
$$

If $p$ is unscanned by the expansion from $R$, we estimate $C_{S D}(p, R) . u b$ according to Equation 7.

$$
C_{S D}(p, R)=\sum_{o \in R} e^{-s d(p, o)}<\sum_{o \in R} e^{-(r-s d(o, c))}=C_{S D}(p, R) \cdot u b
$$

Therefore, we have that

$$
C_{S D}(p, R) \cdot u b= \begin{cases}\sum_{o \in R} e^{-(s d(c, p)-s d(o, c))} & \text { if Case } 1 \\ \sum_{o \in R} e^{-(r-s d(o, c))} & \text { if Case } 2\end{cases}
$$

Case 1: $p$ is scanned by the expansion from $R$.

Case $2: p$ is unscanned by the expansion from $R$.

By substituting Equations 11 into Equation 1, we define the upper bound of the spatial-density correlation of a partly scanned location candidate $p$ as follows.

$$
C_{S D}(p, Q) \cdot u b=\sum_{R_{i} \in Q}\left(\frac{2}{1+e^{-C_{S D}\left(p, R_{i}\right) \cdot u b}}-1\right)
$$

Among all partly scanned location candidates, we define a global upper bound $U B$ of spatial-density correlation as follows. Similar to $L B$, the value of $U B$ changes dynamically during query processing.

$$
U B=\max _{p \in P_{p}} C_{S D}(p, Q) \cdot u b
$$

Here, $P_{p}$ is a set of partly scanned location candidates.

Once the value of $U B$ exceeds that of $L B$, the expansion search terminates, and all partly scanned and unscanned location candidates are pruned. We refine the fully scanned location candidates, and find the query results.

Remark. There is no need to compute and to maintain the upper bound of unscanned location candidates (different to the baseline algorithm). By comparing Equations 7 and 11, for a partly scanned location candidate $p$ and an unscanned location candidate $p^{\prime}$, we have that

$$
C_{S D}(p, R) . u b \geq C_{S D}\left(p^{\prime}, R\right) . u b \Rightarrow C_{S D}(p, Q) . u b>C_{S D}\left(p^{\prime}, Q\right) . u b .
$$

So if $C_{S D}(p, Q) . u b<L B$, we have that $C_{S D}\left(p^{\prime}, Q\right) . u b<L B$. All unscanned location candidates can be pruned safely. 


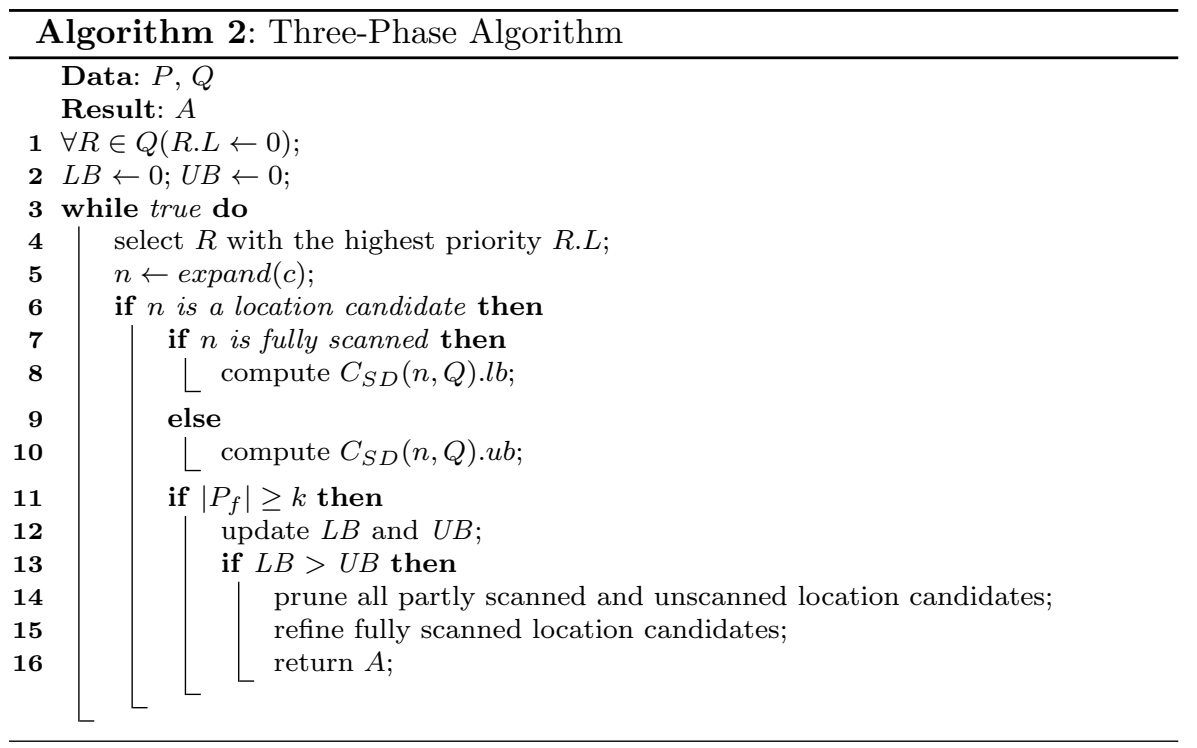

\subsubsection{Scheduling}

To further enhance the query efficiency, we propose a novel heuristic scheduling strategy to schedule the searches from multiple query regions. Each query region is given a label to describe its priority. At each time, we only search the top-ranked query region, until a new top-ranked query region appears. The priority label is defined as follows.

$$
R . L=\sum_{P_{p} \backslash P_{R}}\left\{C_{S D}(p, Q) . u b\right\}
$$

Here, $P_{p}$ is the set of partly scanned location candidates, and $P_{R}$ is a set of location candidates scanned by the search from $R$. Our target is to make partly scanned location candidates to fully scanned as soon as possible. We use $P_{p} \backslash P_{R}$ to describe the gap of region $R$. Moreover, we also consider the upper bound of the spatial-density correlation (see Equation 12) of a location candidate. Obviously, a location candidate with a higher upper bound may have a higher possibility to be the query result. We share the similar idea of existing studies $[24,26,28,29]$.

\subsubsection{Algorithm}

The detailed procedure of the three-phase algorithm is introduced in Algorithm 2. The query arguments include a set $P$ of location candidates and a set $Q$ of query regions. The query result is a set $A(|A|=k \wedge A \subseteq Q)$ of location candidates with largest spatial-density correlations. Initially, the priority labels of all query regions are set to 0 , and the values of global upper 
and lower bounds are set to 0 (lines 1-2). We expand the network expansion according to Dijkstra's algorithm from each query region, and $n$ is a newly scanned vertex (lines $3-5$ ). If $n$ is a location candidate and $n$ is fully scanned, we compute $C_{S D}(n, Q) . l b$ (Equation 6). Otherwise, we compute $C_{S D}(n, Q) . u b$ (Equation 12) (lines 6-10). If the size of fully scanned set exceeds $k$, we compute the global lower bound $L B$ (Equation 9) and global upper bound $U B$ (Equation 13) of fully scanned location candidates (lines 11-12). If the value of $L B$ exceeds that of $U B$, the searches from all query regions terminate, and all partly scanned and unscanned location candidates are pruned. Then, we refine all fully scanned location candidates, and find top- $k$ locations in set $A$ with the largest spatial-density correlations (lines 13-16).

\subsection{Time Complexity Analysis}

Both Algorithms 1 and 2 are based on network expansion (i.e., Dijkstra's algorithm [7]), and their time complexities are $O(|Q|(|V| l g(|V|)+|E|))$, where $|Q|$ is the number of query regions, $|V|$ is the number of vertices and $|E|$ is the number of edges in the corresponding spatial network, and $O(|V| l g(|V|)+|E|)$ is the time complexity of Dijkstra's algorithm. Although Algorithms 1 and 2 have the same time complexity in the worst case, Algorithm 2 has more powerful pruning techniques and it can achieve a higher performance (See the experimental results in Section 4).

\section{Experiments}

\subsection{Settings}

We conduct extensive experiments on real and synthetic spatial data to study the performance of the developed algorithms. We use two spatial networks, called the Beijing Road Network (BRN) and the North America Road Network $(\mathrm{NRN})^{7}$, which contain 28,342 vertices and 27,690 edges, and 17,813 vertices and 179,179 edges, respectively. For BRN, we use 10,000 real POIs as location candidates and use 30,000 real POIs as spatial objects. For NRN, we generate 100,000 location candidates and 400,000 spatial objects. In the following figures, the baseline algorithm (Algorithm 1) is denoted by "baseline", the three-phase algorithm is denoted by "three-phase", and the three-phase algorithm without heuristic scheduling strategy is denoted by "three-phase w-o-h". The algorithms were implemented in Java and performed on a Windows 8 platform (Intel Core 2.90GHz Processor and 8GB memory). The main performance metrics were runtime.

For the parameter setting, the number of location candidates $|P|$ varies from 7,000 to 10,000 in BRN (default 10,000), and varies from 70,000 to 100,000 in NRN (default 100,000). The number of query regions $|Q|$ varies

\footnotetext{
7 http://www.cs.utah.edu/lifeifei/SpatialDataset.htm
} 
Table 1 Parameter settings

\begin{tabular}{|l|l|l|}
\hline & NRN & BRN \\
\hline $\begin{array}{l}\text { Number of Location Can- } \\
\text { didate }|P|\end{array}$ & $70,000-100,000$ (default 100,000) & $7,000-10,000$ (default 10,000) \\
\hline $\begin{array}{l}\text { Number of Query Regions } \\
|Q|\end{array}$ & $20-50$ (default 20) & $10-25$ (default 10) \\
\hline average radius of regions $r$ & $20-80 \mathrm{~km}$ (default 20 km) & $1-4 \mathrm{~km}$ (default 1 km) \\
\hline$k$ & $5-20$ (default 5) & $5-20$ (default 5) \\
\hline
\end{tabular}

from 10 to 25 in BRN (default 10), and varies from 20 to 50 in NRN (default 20). The average radius of regions varies from $1 \mathrm{~km}$ to $4 \mathrm{~km}$ in BRN (default $1 \mathrm{~km}$ ), and varies from $20 \mathrm{~km}$ to $80 \mathrm{~km}$ in NRN (default $20 \mathrm{~km}$ ). The value of $k$ varies from 5 to 20 for both BRN and NRN (default 5). The parameter settings are detailed in Table 1.

4.2 Effect of number of location candidates $|P|$

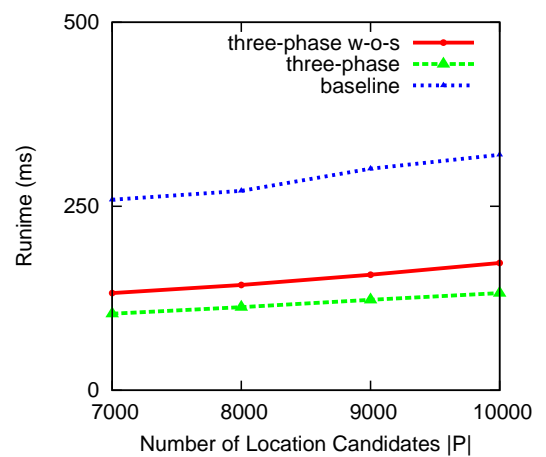

(a) BRN-P

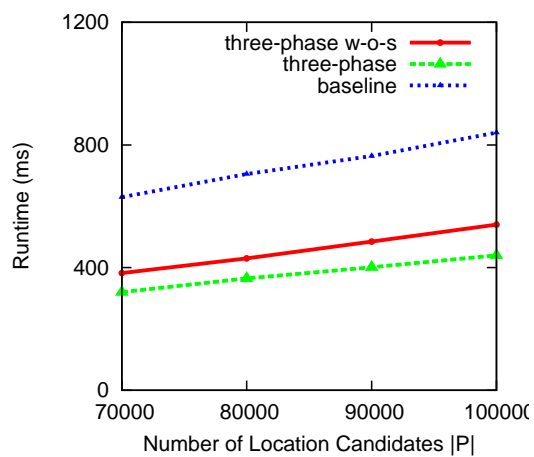

(b) NRN-P

Fig. 4 Effect of number of location candidates $|P|$

First of all, we study the effect of number of location candidates $|P|$ on the $k$ ALDQ query efficiency. Intuitively, a larger number of location candidates $|P|$ may lead to more locations to be processed. Thus, the runtime of all three algorithms are expected to be higher when the number of location candidates $|P|$ increases. From Figure 4, we find that the runtime of the baseline algorithm (Algorithm 1) is about 2-3 times of that of the three-phase algorithm (Algorithm 2). It is clear that the three-phase algorithm has a stronger pruning power so it can achieve a higher query efficiency (the three-phase algorithm prunes partly scanned and unscanned location candidates, while the baseline algorithm only prunes unscanned location candidates). Moreover, we 
notice that with the help of heuristic scheduling strategy (Equation 14), the efficiency of the three-phase algorithm is improved by a factor of $20 \%-30 \%$ in term of runtime, which demonstrates the significant of the scheduling for the scenario of multiple query sources.

4.3 Effect of number of query regions $|Q|$

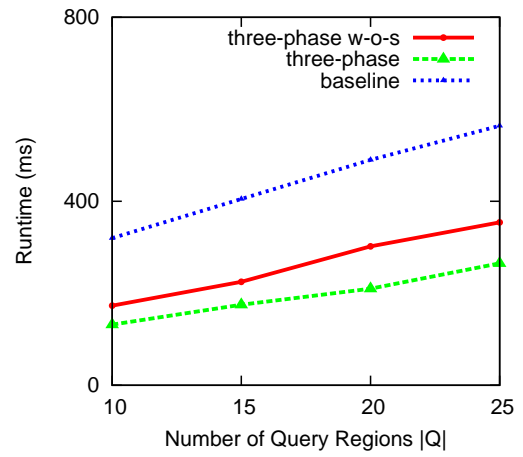

(a) BRN-Q

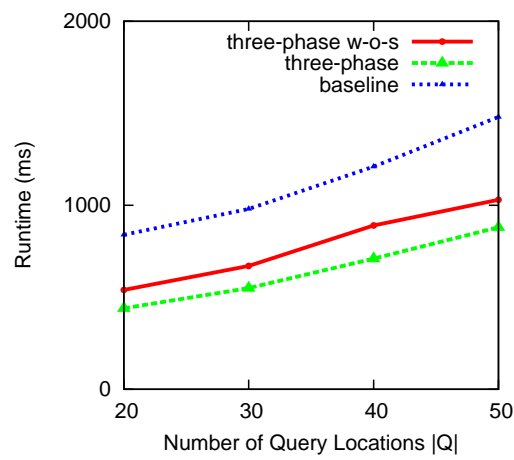

(b) NRN-Q

Fig. 5 Effect of number of query regions $|Q|$

Next, we study the effect of the number of query locations $|Q|$ on the $k$ ALDQ query processing (Algorithms 1 and 2) in BRN and NRN. The $k$ ALDQ query finds the location with the minimum global travel cost to the query regions. Obviously, a larger number of $|Q|$ means more query regions to be refined. For all three algorithms, the larger the number of query regions is, the more computation effort they may need. Thus, the runtime is expected to be higher when the number of query locations $|Q|$ increases. From Figure 5, it is clear that the three-phase algorithm outperforms the baseline algorithm by a factor of 2-3 times in term of runtime, and outperforms the three-phase algorithm without a heuristic strategy by a factor of $20 \%-40 \%$ in term of runtime.

\subsection{Effect of average radius of regions $r$}

Then, we vary the value of the average radius $r$ of query regions. Assuming spatial objects are uniformly distributed. So a larger value of radius means a larger area of a region, and more spatial objects in the region. According to Equations 1 and 2, we see that more spatial objects may need more computation effort. Thus, the runtime of all three algorithm are expected to increase 


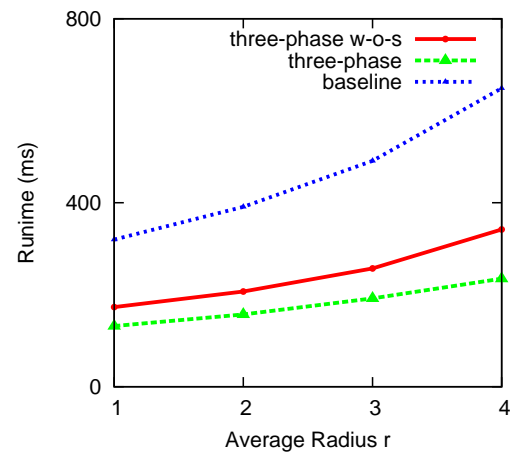

(a) BRN-r

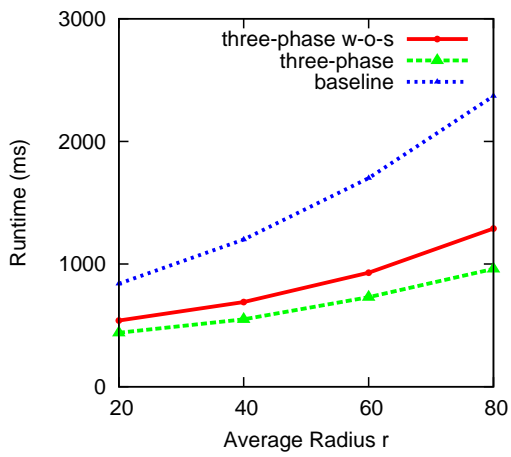

(b) NRN-r

Fig. 6 Effect of average radius of regions $r$

when $r$ increases. Compared to the baseline algorithm, the three-phase algorithm has clear advantage. In Figure 6 , we see that when $r=4$ in $\mathrm{BRN}$, the runtime of baseline is almost three times of that of three-phase algorithm. The three-phase algorithm is able to process query regions with radius of $80 \mathrm{~km}$ in NRN within 1 second.

\subsection{Effect of $k$}

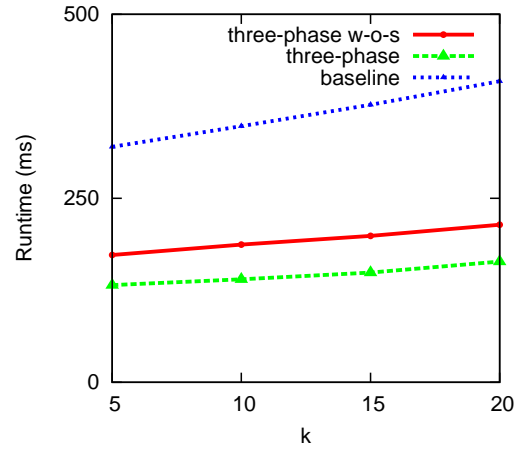

(a) BRN-k

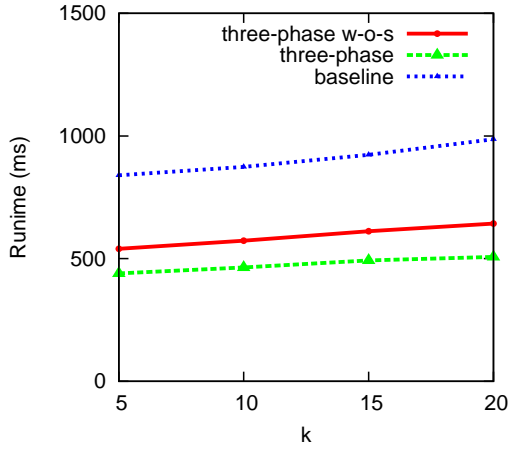

(b) NRN-k

Fig. 7 Effect of $k$

Finally, we study the effect of $k$. We see that a larger value of $k$ may lead to a larger search space and more computation efforts are needed. When the value of $k$ increases, the rumtime of all three algorithm will increase. From 
Figure 7, the three-phase algorithm is able to compute the query within 200 seconds in BRN, and within 500 seconds in NRN. With the help of heuristic scheduling method, the query efficiency is improved notably.

\section{Related Work}

Location search and recommendation are well studied in recent two decades [6, 8-11,19,30,31,43-48]. Generally, such type of query can be classified into three categories: point-to-point, trajectory-to-point, and region-to-point. In the first point-to-point category, the nearest neighbor $(\mathrm{NN})$ query is a basic spatial query. Given a query location and a set of location candidates, NN finds the location candidate with the minimum travel cost to the query location. Aggregate nearest neighbor query (ANN) $[21,22]$ and aggregate location recommendation query (ALRQ) [15] further extend NN query. Given a set of travelers' current locations and a set of potential meeting points, the queries find the optimal meeting point with the minimum global travel cost. These queries are useful in many applications such as travel planning. ANN is based on Euclidean space. In contrast to ANN, ALRQ is conducted in dynamic spatial networks, and the instant traffic conditions are taken into account. Collective travel planning (CTP) [25] query further extends the model of ANN, which finds the travel route with the minimum travel cost from multiple query source to a destination, via $k$ meeting points.

In the trajectory-to-location category, the query find the optimal location based on one or multiple trajectories. In the path nearest neighbor (PNN) query [5,27], the query finds the spatial point with the minimum travel cost to the path. Then, Shang et. al extend the PNN query on compressed trajectories [35], and the uncertainty of trajectory data is taken into account. The reverse path nearest neighbor query [36] uses trajectory data to rank locations, which is useful in finding most accessible location in spatial networks. For example, if a location is the path nearest neighbor of $k$ trajectories, we define its influence factor as $k$.

To the best of our knowledgeless, the top- $k$ accessible location discovery query $(k \mathrm{ALDQ})$ is the first study in the region-to-location category. No existing study considers the regions of interest and the density of spatial objects (e.g., geo-tagged tweets, geo-tagged photos, and POIs) in the corresponding regions. The existing techniques of location discovery and recommendation cannot compute $k$ ALDQ due to three reasons. First, $k$ ALDQ considers the matching between regions and locations, rather than the point-to-point matching or trajectory-to-point matching, thus it needs new distance metrics. Second, $k$ ALDQ takes the density of spatial objects in query regions into account, so it also needs specific metrics to reflect the density domain. Third, most existing studies of aggregate location query $[21,22]$ are conducted in Euclidean space, and their optimization techniques cannot be used in spatial networks directly, because most spatial indexes (e.g., R-tree) use Euclidean space as 
lower bound to prune the search space and such bound is ineffective in spatial networks.

How to use social media data to achieve a better recommendation is an interesting direction. The following related papers [2-4,12-14, 16, 17, 23, 40-42] provide some inspirations. In the future, it is also of interest to study routing problem with geo-social data $[18,20,32-34,38,39,49]$.

\section{Conclusion and Future Studies}

In this paper, we proposed and studied a novel top- $k$ accessible location discovery query ( $k$ ALDQ) in spatial networks. This type of query is useful in many mobile applications, such as travel planning, facility allocation, and urban planning. We develop a range-query based algorithm, and a novel three-phase algorithm with upper and lower bounds and a heuristic scheduling strategy to process the $k$ ALDQ query efficiently. The performance of $k$ ALDQ were verified by extensive experiments on real and synthetic spatial data.

There exist two interesting future directions. First, it is of interest to take into account the significance of query regions. Users can specify significant query regions when conducting the query. So the upper and lower bounds should be reworked. Second, it is of interest to take into account the temporal information (e.g., departure time from a query region) and to make the query a spatiotemporal query.

\section{Acknowledgement}

This paper is partly supported by Natural Science Foundation of P.R.China (No. 61373147, and No. 61672442), Fujian Province Science and Technology Plan Project (No. 2016Y0079, No. 2017J01783), the Education and Scientific Research Project for Youth and Middle-aged Teachers in Fujian (No. JA15365), the Open Research Fund Program of Guangdong Province Key Laboratory of Popular High Performance Computers of Shenzhen University, and the Open Research Fund Program of Guangdong Provincial Big Data Collaborative Innovation Center, Shenzhen University.

\section{References}

1. S. Brakatsoulas, D. Pfoser, R. Salas, and C. Wenk. On map-matching vehicle tracking data. In $V L D B$, pages 853-864, 2005.

2. Z. Chen, M. Cafarella, J. Chen, D. Prevo, and J. Zhuang. Senbazuru: A prototype spreadsheet database management system. PVLDB, 6(12):1202-1205, 2013.

3. Z. Chen and M. J. Cafarella. Integrating spreadsheet data via accurate and low-effort extraction. In SIGKDD, pages 1126-1135, 2014.

4. Z. Chen, M. J. Cafarella, and H. V. Jagadish. Long-tail vocabulary dictionary extraction from the web. In WSDM, pages 625-634, 2016.

5. Z. Chen, H. Shen, X. Zhou, Y. Zheng, and X. Xie. Searching trajectories by locations: an efficiency study. In SIGMOD, pages 255-266. ACM, 2010. 
6. J. Dai, B. Yang, C. Guo, C. S. Jensen, and J. Hu. Path cost distribution estimation using trajectory data. $P V L D B, 10(3), 2017$.

7. E. W. Dijkstra. A note on two problems in connection with graphs. Numerische Math, 1:269-271, 1959.

8. Z. Ding, B. Yang, Y. Chi, and L. Guo. Enabling smart transportation systems: A parallel spatio-temporal database approach. IEEE Trans. Computers, 65(5):1377-1391, 2016.

9. Z. Ding, B. Yang, R. H. Güting, and Y. Li. Network-matched trajectory-based movingobject database: Models and applications. IEEE Trans. Intelligent Transportation Systems, 16(4):1918-1928, 2015.

10. C. Guo, C. S. Jensen, and B. Yang. Towards total traffic awareness. SIGMOD Record, 43(3):18-23, 2014.

11. C. Guo, B. Yang, O. Andersen, C. S. Jensen, and K. Torp. Ecosky: Reducing vehicular environmental impact through eco-routing. In ICDE, pages 1412-1415, 2015.

12. D. Guo, Y. Zhu, W. Xu, S. Shang, and Z. Ding. How to find appropriate automobile exhibition halls: Towards a personalized recommendation service for auto show. Neurocomputing, 213:95-101, 2016.

13. J. Han, K. Zheng, A. Sun, S. Shang, and J. Wen. Discovering neighborhood pattern queries by sample answers in knowledge base. In ICDE, pages 1014-1025, 2016.

14. S. Hu, J. Wen, Z. Dou, and S. Shang. Following the dynamic block on the web. World Wide Web, 19(6):1077-1101, 2016.

15. J. Li, Y. Wang, Y. Zhong, D. Guo, and S. Zhu. Aggregate location recommendation in dynamic transportation networks. World Wide Web Journal, online first:1-18, 2017.

16. A. Liu, W. Wang, S. Shang, Q. Li, and X. Zhang. Efficient task assignment in spatial crowdsourcing with worker and task privacy protection. online first:1-26, 2017.

17. J. Liu, S. Shang, K. Zheng, and J. Wen. Multi-view ensemble learning for dementia diagnosis from neuroimaging: An artificial neural network approach. Neurocomputing, 195:112-116, 2016

18. J. Liu, K. Zhao, P. Sommer, S. Shang, B. Kusy, J. Lee, and R. Jurdak. A novel framework for online amnesic trajectory compression in resource-constrained environments. IEEE Trans. Knowl. Data Eng., 28(11):2827-2841, 2016.

19. K. Liu, Y. Li, Z. Ding, S. Shang, and K. Zheng. Benchmarking big data for trip recommendation. In $I C C C N$, pages 1-6, 2014.

20. K. Liu, B. Yang, S. Shang, Y. Li, and Z. Ding. MOIR/UOTS: trip recommendation with user oriented trajectory search. In $M D M$, pages 335-337, 2013.

21. D. Papadias, Q. Shen, Y. Tao, and K. Mouratidis. Group nearest neighbor queries. In ICDE, pages 301-312, 2004.

22. D. Papadias, Y. Tao, K. Mouratidis, and C. K. Hui. Aggregate nearest neighbor queries in spatial databases. TODS, 30(2):529-576, 2005.

23. X. Rong, Z. Chen, Q. Mei, and E. Adar. Egoset: Exploiting word ego-networks and user-generated ontology for multifaceted set expansion. In WSDM, pages 645-654, 2016.

24. S. Shang, L. Chen, C. S. Jensen, J. Wen, and P. Kalnis. Searching trajectories by regions of interest. IEEE Trans. Knowl. Data Eng., 29(7):1549-1562, 2017.

25. S. Shang, L. Chen, Z. Wei, C. S. Jensen, J. Wen, and P. Kalnis. Collective travel planning in spatial networks. IEEE Trans. Knowl. Data Eng., 28(5):1132-1146, 2016.

26. S. Shang, L. Chen, Z. Wei, C. S. Jensen, K. Zheng, and P. Kalnis. Trajectory similarity join in spatial networks. PVLDB, 10(11):1178-1189, 2017.

27. S. Shang, K. Deng, and K. Xie. Best point detour query in road networks. In $A C M$ GIS, pages 71-80, 2010.

28. S. Shang, R. Ding, B. Yuan, K. Xie, K. Zheng, and P. Kalnis. User oriented trajectory search for trip recommendation. In EDBT, pages 156-167, 2012.

29. S. Shang, R. Ding, K. Zheng, C. S. Jensen, P. Kalnis, and X. Zhou. Personalized trajectory matching in spatial networks. $V L D B$ J., 23(3):449-468, 2014.

30. S. Shang, D. Guo, J. Liu, and K. Liu. Human mobility prediction and unobstructed route planning in public transport networks. In $M D M(2)$, pages 43-48, 2014.

31. S. Shang, D. Guo, J. Liu, K. Zheng, and J. Wen. Finding regions of interest using location based social media. Neurocomputing, 173:118-123, 2016.

32. S. Shang, J. Liu, K. Zheng, H. Lu, T. B. Pedersen, and J. Wen. Planning unobstructed paths in traffic-aware spatial networks. GeoInformatica, 19(4):723-746, 2015. 
33. S. Shang, H. Lu, T. B. Pedersen, and X. Xie. Finding traffic-aware fastest paths in spatial networks. In $S S T D$, pages 128-145, 2013.

34. S. Shang, H. Lu, T. B. Pedersen, and X. Xie. Modeling of traffic-aware travel time in spatial networks. In MDM (1), pages 247-250, 2013.

35. S. Shang, B. Yuan, K. Deng, K. Xie, K. Zheng, and X. Zhou. Pnn query processing on compressed trajectories. GeoInformatica, 16(3):467-496, 2012.

36. S. Shang, B. Yuan, K. Deng, K. Xie, and X. Zhou. Finding the most accessible locations: reverse path nearest neighbor query in road networks. In ACM SIGSPATIAL, pages 181-190, 2011.

37. S. Shang, K. Zheng, C. S. Jensen, B. Yang, P. Kalnis, G. Li, and J. Wen. Discovery of path nearby clusters in spatial networks. IEEE Trans. Knowl. Data Eng., 27(6):15051518, 2015.

38. S. Shang, S. Zhu, D. Guo, and M. Lu. Discovery of probabilistic nearest neighbors in traffic-aware spatial networks. World Wide Web, 20(5):1135-1151, 2017.

39. K. Xie, K. Deng, S. Shang, X. Zhou, and K. Zheng. Finding alternative shortest paths in spatial networks. ACM Trans. Database Syst., 37(4):29:1-29:31, 2012.

40. Q. Xie, S. Shang, B. Yuan, C. Pang, and X. Zhang. Local correlation detection with linearity enhancement in streaming data. In CIKM, pages 309-318, 2013.

41. X. Xie, H. Lu, J. Chen, and S. Shang. Top-k neighborhood dominating query. In DASFAA, pages 131-145, 2013

42. Y. Xu, L. Chen, B. Yao, S. Shang, S. Zhu, K. Zheng, and F. Li. Location-based top-k term querying over sliding window. In WISE, pages 299-314, 2017.

43. B. Yang, J. Dai, C. Guo, and C. S. Jensen. Pace: A PAth-CEntric paradigm for stochastic path finding. VLDB Journal, online first, 2015.

44. B. Yang, C. Guo, C. S. Jensen, M. Kaul, and S. Shang. Stochastic skyline route planning under time-varying uncertainty. In $I C D E$, pages 136-147, 2014

45. K. Zheng, S. Shang, N. J. Yuan, and Y. Yang. Towards efficient search for activity trajectories. In ICDE, pages 230-241, 2013.

46. K. Zheng, H. Su, B. Zheng, S. Shang, J. Xu, J. Liu, and X. Zhou. Interactive top-k spatial keyword queries. In ICDE, pages 423-434, 2015.

47. K. Zheng, Y. Zheng, N. J. Yuan, and S. Shang. On discovery of gathering patterns from trajectories. In ICDE, pages 242-253, 2013.

48. K. Zheng, Y. Zheng, N. J. Yuan, S. Shang, and X. Zhou. Online discovery of gathering patterns over trajectories. IEEE Trans. Knowl. Data Eng., 26(8):1974-1988, 2014.

49. S. Zhu, Y. Wang, S. Shang, G. Zhao, and J. Wang. Probabilistic routing using multimodal data. Neurocomputing, 253:49-55, 2017. 